\title{
Cross-compatibility in Intraspecific and Interspecific Hybridization within Lychnis and Intergeneric Hybridization between Lychnis and Silene
}

\author{
Li Jiang ${ }^{1}$ \\ Department of Horticulture and Landscape Architecture, Oklahoma State \\ University, Stillwater, OK 74078-6027
}

Yun-wen Wang

Department of Grassland Science, College of Animal Science and Technology, China Agricultural University, Beijing 100193, The People's Republic of China

\author{
Bruce L. Dunn ${ }^{2}$ \\ Department of Horticulture and Landscape Architecture, Oklahoma State \\ University, $358 \mathrm{Ag} \mathrm{Hall,} \mathrm{Stillwater,} \mathrm{OK} \mathrm{74078-6027}$
}

Additional index words. Caryophyllaceae, ornamental breeding, seed morphology, seed set

\begin{abstract}
Hybridization at intraspecific, interspecific, and intergeneric levels is a wellknown breeding strategy to create variations with desirable traits for ornamental flowers. A total of 161 crossing combinations were made on three taxonomic levels, including 12 intraspecific crossing combinations within $L$. chalcedonica and $L . \times$ haageana, 102 interspecific crossing combinations within Lychnis spp., and 47 intergeneric crossing combinations between Lychnis spp. and Silene spp. Intraspecific crosses showed high cross-compatibility, which yielded mature seeds and progeny plants. Most of the interspecific crossings in genus Lychnis produced limited seed set and germination percentages; however, L. cognate, $L$. $\times$ arkwrightii, and $L$. $\times$ haageana showed high crosscompatibility and might be more closely related than other species in Lychnis. As a result of cross-incompatibility, crossing combinations between Lychnis spp. and Silene spp. produced few flowers that set seeds. Significant differences occurred in seed set between crossing combinations and their reciprocal crosses for interspecific and intergeneric crosses. For these hybrids with immature seeds, embryo rescue techniques with immature seed culture would be the only way to produce seedlings.
\end{abstract}

The genera Lychnis L. and Silene L. belong to the family Caryophyllaceae. Lychnis species, commonly known as campion or catchfly, are native to the temperate regions of the Northern Hemisphere, from East Asia to Central Asia, Europe, and North and East Africa (Oxelman et al., 2000; Popp et al., 2008). Lychnis is characterized by perennial herbs, consisting of $\approx 30$ species with some species being extensively cultivated as landscape ornamentals. Silene is the largest genus in Caryophyllaceae, consisting of biennial or perennial herbs with species numbers varying from 500 to 700 (Melzheimer, 1988; Oxelman et al., 2000; Walters, 1989). Silene species occur worldwide, although the greatest diversity occurs in the Mediterranean region (Oxelman et al., 2000). The taxonomic boundary between Lychnis and Silene L. is

\footnotetext{
Received for publication 28 Apr. 2014. Accepted for publication 24 July 2014

${ }^{1}$ Former graduate student. Currently a doctoral student, Department of Plant and Environmental Sciences, New Mexico State University.

${ }^{2}$ To whom reprint requests should be addressed; e-mail bruce.dunn@okstate.edu.
}

often disputed (Desfeux and Lejeune, 1996; Kruckeberg, 1962). Both genera have been merged in cladistic theory (Desfeux and Lejeune, 1996; Negrutiu et al., 2001), yet Lychnis as an independent genus is also accepted by researchers beyond taxonomic fields. Distinction between the traditional genera Lychnis and Silene in morphology is that Lychnis usually has five styles and an entire capsule, whereas Silene has three styles and a split capsule (Desfeux and Lejeune, 1996).

Lychnis has ornamental merit because most species are perennial, have good drought tolerance, and exhibit high diversity in morphology and ecophysiology. The majority of Lychnis and Silene species have identical somatic chromosome numbers $(2 \mathrm{n}=2 \mathrm{x}=24)$ (Negrutiu et al., 2001), which suggests the possibility of hybridization for exploring desirable and exotic hybrids for commercial use. Hybridization has been used to test the phylogeny relationship of Lychnis and Silene (Kruckeberg, 1962; Wilson et al., 1995). A number of cultivars are commercially available and were obtained from intraspecific breeding (Godo et al., 2009;
Mayol and Rossello, 2006). An exceptional example is Lychnis xarkwrightii Heydt, which is a putative hybrid between Lychnis chalcedonica L. and Lychnis Xhaageana Lemoine. The majority of Lychnis species are hermaphrodites and are cross-pollinated by insects, which is aided by being protandrous, although self-pollination does occur. Therefore, crossing combinations were made to test the crossability among Lychnis and Silene species and cultivars.

\section{Materials and Methods}

Plant materials and growth conditions. Seven Lychnis species, totaling 20 accessions including commercial cultivars, hybrids, and species, and five Silene species were used for hybridizations (Table 1). A total of 161 crossing combinations were made on three taxonomic levels including: 1) 12 intraspecific crossing combinations within L. chalcedonica and L. Xhaageana; 2) 102 interspecific crossing combinations within Lychnis spp.; and 3) 47 intergeneric crossing combinations between Lychnis spp. and Silene spp. Five crosses including reciprocal crosses were attempted for each combination without emasculation, yet the actual crossing numbers were restricted to flower availability. Overall, 49 crosses were made for intraspecific crossing, 623 crosses were made for interspecific crossing, and 290 crosses were made for intergeneric crossing. A total of 962 crosses were made for all combinations. To facilitate the large number of hybridizations, flowers were not emasculated; however, all progeny were evaluated based on flower and plant morphological traits to determine if progeny was the result of selfing. Progeny was removed from the study if they were a result of selfing. Fertilized capsules were collected as the color changed from green to brown. All hybridizations were conducted during May to Oct. 2010 in the Department of Horticulture and Landscape Architecture Research Greenhouses, Oklahoma State University, Stillwater, OK, under natural photoperiods. Temperature was set at $18 / 21{ }^{\circ} \mathrm{C}$ day/night with a photosynthetic photon flux density range of 600 to $1200 \mu \mathrm{mol} \cdot \mathrm{m}^{-2} \cdot \mathrm{s}^{-1}$ at $1200 \mathrm{HR}$. Seeds from individual capsules were sown in Metro-mix 702 (Sun Gro Horticulture, Bellevue, WA) media placed in $15-\mathrm{cm}$ round pots (Itml, Middlefield, OH) starting July 2010 in the greenhouse.

Data collection. Beginning in July 2010, the number of crosses, number of successful crosses, $F_{1}$ seed number, and germination rates were recorded. Phenotypic traits including leaf color, flower color, and size were recorded starting in May 2011. Hybrid flower morphological data of corolla diameter, petal width, and petal length were recorded on five flowers, each of them from an individual plant. Flower and leaf color were recorded using The Royal Horticultural Society Color Charts (1966). All hybrid plants were cultivated in the greenhouse when the color data were taken. The traits were analyzed using 
analysis of variance (PROC GLM, Version 9.3; $\mathrm{SAS}^{\circledR}$, Cary, NC), and means were compared using a Duncan test $(P \leq 0.05$, $\mathrm{n}=5$ ).

\section{Results}

Intraspecific crosses. All 12 of the intraspecific combinations within Lychnis species set seed (Tables 2 and 3). Among six accessions within L. chalcedonica and 48 pollinated flowers, on average $83.3 \%$ set seed and had an average seed number of 52 per capsule. A total of 2094 seeds was collected from intraspecific crosses within L. chalcedonica, and average germination rate was $64.7 \%$ (Table 2). Only one intraspecific cross between $L$. Xhaageana 'Molten Lava' and L. Xhaageana mixed hybrids was made, of which 24 seeds were collected from the single capsule and $91.7 \%$ of them germi- nated normally (Tables 2 and 3 ). These results confirmed that interbreeding within species could successfully produce fertile seed and progeny. For L. chalcedonica, L. chalcedonica var. alba, and $L$. chalcedonica 'White', differences in percent of flowers that set seeds and seed germination rates within reciprocal crosses occurred (Tables 2 and 3). In particular, for a cross combination between L. chalcedonica 'White' and L. chalcedonica, seed germination rate was $84.8 \%$ when L. chalcedonica 'White' as a maternal parent, much higher than that of $22.0 \%$ as a paternal parent. Red flower was dominant in all intraspecific $\mathrm{F}_{1}$ hybrid plants among $L$. chalcedonica (red), L. chalcedonica var. alba, L. chalcedonica 'White', and L. chalcedonica 'Rauhreif' (white), or L. chalcedonica 'Carnea' (dusty pink) flowers (data not shown).

Interspecific crosses. Compared with the intraspecific crosses, most interspecific

Table 1. Lychnis and Silene species and cultivars used in hybridization and their seed sources.

\author{
Species and cultivars \\ Lychnis \\ L. chalcedonica \\ L. chalcedonica var. alba \\ L. chalcedonica 'White' \\ L. chalcedonica 'Rauhreif' \\ L. chalcedonica 'Carnea' \\ L. chalcedonica 'Burning Love' \\ L. cognata Maxim. \\ L. miqueliana Rohrb. \\ L. wilfordii (Regel) Maxim. \\ L. flos-cuculi L. \\ L. flos-cuculi 'White Robin' \\ L. Xhaageana mixed hybrids \\ L. Xhaageana 'Molten Lava' \\ L. ×haageana 'Lumina Salmon' \\ L. Xhaageana 'Lumina Orange' \\ L. Xhaageana 'Lumina Bronze Leaf Red' \\ L. ×arkwrightii \\ L. arkwrightii 'Vesuvius' \\ L. xarkwrightii 'Orange Genome'
} Seed sources

\author{
J.L. Hudson, Seedsman, CA \\ Chiltern Seeds, Wallingford, UK \\ Hardyplants.com, MN \\ Hardyplants.com, MN \\ Hardyplants.com, MN \\ Hardyplants.com, MN \\ B \& T World Seeds, Paguignan, France \\ Arrowhead Alpines-Bob \& Brigitta Stewart, MI \\ Arrowhead Alpines-Bob \& Brigitta Stewart, MI \\ J.L. Hudson, Seedsman, CA \\ Hardyplants.com, MN \\ Chiltern Seeds, Wallingford, UK \\ Seeds-by-Size, Hemel Hempstead, UK \\ Hardyplants.com, MN \\ Hardyplants.com, MN \\ Hardyplants.com, MN \\ B \& T World Seeds, Paguignan, France \\ J.L. Hudson, Seedsman, CA \\ Hardyplants.com, MN
}

Silene

S. armeria $\mathrm{L}$.

S. pendula L.

S. plankii C.L. Hitchc. \& Maguire

S. acaulis ssp. Acaulescens (F.N. Williams) C.L.

Hitchc. \& Maguire

S. delavayi Franch.

B \& T World Seeds, Paguignan, France

B \& T World Seeds, Paguignan, France

Alplains, $\mathrm{CO}$

B \& T World Seeds, Paguignan, France

B \& T World Seeds, Paguignan, France crosses in the genus Lychnis produced fewer flowers that set seed and had lower seed germination percentages (Tables 4 and 5). However, the percent of flowers that set seed and germinated was relatively high for the crossing pairs between $L$. cognata and $L$. xarkwrightii 'Vesuvius', and L. ×haageana (L. Xhaageana mixed hybrids and 'Molten Lava', 'Lumina Orange', and 'Lumina Broze leaf Red'), and also between L. ×arkwrightii 'Vesuvius' and L. Xhaageana mixed hybrids. In the crossing pairs where L. cognata was the maternal parent, $57.1 \%$ of crosses with L. Xarkwrightii 'Vesuvius' and $80.0 \%$ of crosses with $L$. Xhaageana mixed hybrids produced seed and seed germination rates were $77.1 \%$ and $68.5 \%$, respectively (Table 6 ). In crosses with $L$. $\times$ arkwrightii 'Vesuvius' as the maternal parent and $L$. cognate as the pollen parent, seed set and seed germination rates were $93.8 \%$ and $69.2 \%$, respectively. Seed set was lower for the crossing pairs with $L$. Xhaageana mixed hybrids where L. cognate was the pollen parent; however, seed germination rates were similar (Table 6). In the reciprocal crosses between $L$. Xhaageana 'Molten Lava' and L. cognate, and the crosses between L. Xhaageana 'Lumina Orange' or 'Lumina Bronze Leaf Red' with L. cognate, $100.0 \%$ of crosses produced seeds, and seed germination rates ranged from $53.2 \%$ to $87.3 \%$ (Table 6). For L. xarkwrightii 'Vesuvius' $\times$ L. Xhaageana mixed hybrids, $40.0 \%$ of pollinated flowers set seed and $60.7 \%$ of the seeds germinated. Meanwhile, the reciprocal crosses between the two accessions yielded $87.5 \%$ seed setting flowers and $68.2 \%$ seed germination (Table 6). The results demonstrated that no interbreeding barrier exists within the three species including $L$. cognate, $L$. $\times$ arkwrightii, and $L$. $\times$ haageana out of the seven species we tested in genus Lychnis. They are likely more closely related to each other than the other species.

For the other 403 interspecific crossing pairs in Lychnis, except for L. miqueliana $\times L$. xarkwrightii 'Orange Genome', all of the interspecific crossing pairs produced seeds (Tables 4 and 5). Considering the average

Table 2. Flower pollination, seed set, and germination for intraspecific crosses as seed parent within Lychnis.

\begin{tabular}{|c|c|c|c|c|c|c|}
\hline Seed parent & $\begin{array}{l}\text { No. of cultivars } \\
\text { mating }\end{array}$ & $\begin{array}{c}\text { No. of cross-pollinated } \\
\text { flowers }\end{array}$ & $\begin{array}{l}\text { Percent of flowers } \\
\text { setting seed }\end{array}$ & $\begin{array}{l}\text { Avg seed no. } \\
\text { per capsule }\end{array}$ & $\begin{array}{l}\text { Total no. } \\
\text { of seeds }\end{array}$ & $\begin{array}{c}\text { Percent } \\
\text { germination }\end{array}$ \\
\hline L. chalcedonica & 3 & 16 & 93.8 & 52 & 786 & 55.7 \\
\hline L. chalcedonica 'Rauhreif' & 2 & 5 & 100.0 & 66 & 329 & 38.9 \\
\hline L. chalcedonica 'White' & 3 & 16 & 81.3 & 53 & 686 & 85.0 \\
\hline L. chalcedonica var. alba & 3 & 11 & 63.6 & 42 & 293 & 70.3 \\
\hline Total for L. chalcedonica & 6 & 48 & 83.3 & 52 & 2094 & 64.7 \\
\hline L. ×haageana 'Molten Lava' & 1 & 1 & 100.0 & 24 & 24 & 91.7 \\
\hline
\end{tabular}

Table 3. Flower pollination, seed set, and germination for intraspecific crosses as pollen parent within Lychnis.

\begin{tabular}{|c|c|c|c|c|c|c|}
\hline Pollen parent & $\begin{array}{l}\text { No. of cultivars } \\
\text { mating }\end{array}$ & $\begin{array}{l}\text { No. of cross-pollinated } \\
\text { flowers }\end{array}$ & $\begin{array}{l}\text { Percent of flowers } \\
\text { setting seed }\end{array}$ & $\begin{array}{l}\text { Avg seed no. } \\
\text { per capsule }\end{array}$ & $\begin{array}{l}\text { Total no. } \\
\text { of seeds }\end{array}$ & $\begin{array}{c}\text { Percent } \\
\text { germination }\end{array}$ \\
\hline L. chalcedonica 'White' & 1 & 5 & 100.0 & 62 & 309 & 22.0 \\
\hline L. chalcedonica var. alba & 1 & 5 & 80.0 & 41 & 162 & 77.8 \\
\hline L. chalcedonica 'Carnea' & 4 & 14 & 92.9 & 46 & 604 & 73.5 \\
\hline L. Xhaageana mixed hybrids & 1 & 1 & 100.0 & 24 & 24 & 91.7 \\
\hline
\end{tabular}


Table 4. Flower pollination, seed set, and germination for interspecific crosses excluding crosses in Table 6 as the seed parent within Lychnis.

\begin{tabular}{|c|c|c|c|c|c|c|c|}
\hline Seed parent & $\begin{array}{l}\text { No. of species } \\
\text { mating }\end{array}$ & $\begin{array}{l}\text { No. of cultivars } \\
\text { mating }\end{array}$ & $\begin{array}{c}\text { No. of cross- } \\
\text { pollinated flowers }\end{array}$ & $\begin{array}{l}\text { Percent of flowers } \\
\text { setting seed }\end{array}$ & $\begin{array}{l}\text { Avg seed no. } \\
\text { per capsule }\end{array}$ & $\begin{array}{l}\text { Total no. } \\
\text { of seeds }\end{array}$ & $\begin{array}{c}\text { Percent } \\
\text { germination }\end{array}$ \\
\hline L. chalcedonica 'Burning Love' & 2 & 2 & 10 & 20.0 & 25 & 50 & 0.0 \\
\hline L. chalcedonica 'Carnea' & 3 & 3 & 18 & 50.0 & 32 & 288 & 0.0 \\
\hline L. chalcedonica 'White' & 5 & 5 & 40 & 17.5 & 15 & 102 & 0.0 \\
\hline L. chalcedonica var. alba & 4 & 4 & 47 & 8.5 & 25 & 100 & 1.0 \\
\hline Total for L. chalcedonica & 5 & 7 & 178 & 25.8 & 31 & 1443 & 0.07 \\
\hline L. ×arkwrightii 'Orange Genome' & 3 & 3 & 7 & 28.6 & 39 & 77 & 0.0 \\
\hline Total for L. ×arkwrightii & 6 & 12 & 76 & 61.8 & 28 & 1304 & 0.0 \\
\hline L. flos-cuculi & 4 & 4 & 30 & 6.7 & 2 & 4 & 0.0 \\
\hline L. flos-cuculi 'White Robin' & 3 & 4 & 21 & 9.5 & 3 & 6 & 0.0 \\
\hline Total for L. flos-cuculi & 5 & 6 & 51 & 7.8 & 3 & 10 & 0.0 \\
\hline L. cognata & 4 & 8 & 36 & 33.3 & 21 & 249 & 0.0 \\
\hline L. miqueliana & 5 & 13 & 45 & 46.7 & 31 & 660 & 0.0 \\
\hline L. wilfordii & 6 & 9 & 72 & 55.6 & 15 & 607 & 0.0 \\
\hline
\end{tabular}

Table 5. Flower pollination, seed set, and germination for interspecific crosses excluding crosses in Table 6 as the pollen parent within Lychnis.

\begin{tabular}{|c|c|c|c|c|c|c|c|}
\hline Pollen parent & $\begin{array}{l}\text { No. of species } \\
\text { mating }\end{array}$ & $\begin{array}{l}\text { No. of cultivars } \\
\text { mating }\end{array}$ & $\begin{array}{c}\text { No. of cross- } \\
\text { pollinated flowers }\end{array}$ & $\begin{array}{l}\text { Percent of flowers } \\
\text { setting seed }\end{array}$ & $\begin{array}{l}\text { Avg seed no. } \\
\text { per capsule }\end{array}$ & $\begin{array}{l}\text { Total no. } \\
\text { of seeds }\end{array}$ & $\begin{array}{c}\text { Percent } \\
\text { germination }\end{array}$ \\
\hline L. chalcedonica 'Burning Love' & 2 & 2 & 2 & 50.0 & 7 & 7 & 0.0 \\
\hline L. chalcedonica 'Carnea' & 3 & 4 & 19 & 63.2 & 27 & 321 & 0.0 \\
\hline L. chalcedonica 'White' & 5 & 6 & 20 & 70.0 & 23 & 320 & 0.0 \\
\hline L. chalcedonica var. alba & 5 & 6 & 30 & 70.0 & 17 & 354 & 0.3 \\
\hline Total for L. chalcedonica & 5 & 9 & 135 & 64.3 & 30 & 2348 & 0.04 \\
\hline L. Xarkwrightii 'Vesuvius' & 5 & 10 & 72 & 36.1 & 17 & 448 & 0.0 \\
\hline L. ×arkwrightii 'Orange Genome' & 1 & 1 & 1 & 0.0 & 0 & 0 & 0.0 \\
\hline Total for L. ×arkwrightii & 6 & 11 & 75 & 37.3 & 19 & 535 & 0.0 \\
\hline L. flos-cuculi & 4 & 6 & 26 & 11.5 & 9 & 27 & 0.0 \\
\hline L. flos-cuculi 'White Robin' & 2 & 3 & 10 & 30.0 & 7 & 21 & 0.0 \\
\hline Total for L. flos-cuculi & 4 & 6 & 36 & 16.7 & 8 & 48 & 0.0 \\
\hline L. cognata & 6 & 11 & 60 & 35.0 & 30 & 635 & 0.0 \\
\hline L. miqueliana & 6 & 14 & 118 & 41.5 & 24 & 1177 & 0.3 \\
\hline L. wilfordii & 6 & 7 & 31 & 12.9 & 10 & 38 & 0.0 \\
\hline
\end{tabular}

data for each species, L. flos-cuculi and $L$. wilfordii produced relatively few seed setting flowers, seeds per capsule, and number of germinated seeds. The results indicated that some interspecific cross-compatibility barriers prevented hybridization and obtaining normal seeds from crossing combinations including $L$. chalcedonica $\times L$. cognata, $L$. chalcedonica $\times$ L. miqueliana, L. chalcedonica $\times$ L. $\times$ arkwrightii, $L$. chalcedonica $\times L$. $\times$ haageana, L. wilfordii $\times$ L. chalcedonica, L. cognate $\times$ L. flos-cuculi, L. cognate $\times L$. miqueliana, $L$. cognate $\times L$. wilfordii, $L$. miqueliana $\times L$. wilfordii, L. miqueliana $\times L$. $\times$ arkwrightii, L. flos-cuculi $\times$ L. miqueliana, $L$. miqueliana $\times$ L. $\times$ haageana, L. flos-cuculi $\times$ L. $\times$ arkwrightii, L. wilfordii $\times$ L. $\times$ arkwrightii, $L$. wilfordii $\times L$. $\times$ haageana, $L$. wilfordii $\times L$. flos-cuculi, and L. flos-cuculi $\times$ L. $\times$ haageana. Nevertheless, because most of the crossing combinations produced immature seeds, embryo rescue techniques with immature seed culture might overcome the barriers and produce hybrid seedlings.

For the interspecific crosses among Lychnis species, there were significant differences for seed set between crossing combinations and their reciprocal crosses (Tables 4, 5 , and 6). Therefore, crossing two interspecific parents in both directions would be a practical method to partially overcome cross-incompatibility between two species in Lychnis. $\mathrm{F}_{1}$ hybrid plants from 11 cross-compatible combinations among $L$. cognate, L. Xarkwrightii, L. ×haageana, and from $L$. Xhaageana 'Molten Lava' $\times L$. miqueliana had intermediate ornamental traits like flower color, stem color, stem hardness, and leaf color between both parents (Tables 7 and 8). These phenotypes showed that promising hybrid plants could be obtained from interspecific crosses, although their hybrid identities need to be evaluated further by molecular marker methods or by observing segregation patterns in $\mathrm{F}_{2}$ generation. For $L$. $\times$ arkwrightii 'Vesuvius' $\times L$. chalcedonica var. alba, L. chalcedonica var. alba $\times$ L. $\times$ haageana mixed hybrids, 
Table 6. Flower pollination, seed set, and germination for interspecific crosses between Lychnis cognata and L. ×arkwrightii, or L. $\times$ haageana.

\begin{tabular}{|c|c|c|c|c|c|c|}
\hline Seed parent & Pollen parent & $\begin{array}{l}\text { No. of cross- } \\
\text { pollinated flowers }\end{array}$ & $\begin{array}{l}\text { Percent of flowers } \\
\text { setting seed }\end{array}$ & $\begin{array}{l}\text { Avg seed no. } \\
\text { per capsule }\end{array}$ & $\begin{array}{l}\text { Total no. } \\
\text { of seeds }\end{array}$ & $\begin{array}{c}\text { Percent } \\
\text { germination }\end{array}$ \\
\hline L. cognata & L. ×arkwrightii 'Vesuvius' & 7 & 57.1 & 27 & 109 & 77.1 \\
\hline L. cognata & L. $\times$ haageana mixed hybrids & 10 & 80.0 & 42 & 292 & 68.5 \\
\hline L. cognata & L. ×haageana 'Molten Lava' & 5 & 100.0 & 19 & 77 & 53.2 \\
\hline L. ×arkwrightii 'Vesuvius' & L. cognata & 16 & 93.8 & 44 & 656 & 69.2 \\
\hline L. $\times$ haageana mixed hybrids & L. cognata & 20 & 60.0 & 42 & 458 & 75.3 \\
\hline L. ×haageana 'Molten Lava' & L. cognata & 4 & 100.0 & 68 & 204 & 87.3 \\
\hline L. ×haageana 'Lumina Orange' & L. cognata & 1 & 100.0 & 25 & 25 & 68.0 \\
\hline $\begin{array}{l}\text { L. ×haageana 'Lumina Bronze } \\
\text { Leaf Red' }\end{array}$ & L. cognata & 1 & 100.0 & 11 & 11 & 64.0 \\
\hline L. ×arkwrightii 'Vesuvius' & L. $\times$ haageana mixed hybrids & 5 & 40.0 & 42 & 84 & 60.7 \\
\hline L. $\times$ haageana mixed hybrids & L. ×arkwrightii 'Vesuvius' & 8 & 87.5 & 39 & 274 & 68.2 \\
\hline
\end{tabular}

Table 7. Flower color in parents and their hybrids for interspecific crosses in Lychnis.

\begin{tabular}{|c|c|c|c|c|}
\hline Maternal color ${ }^{2}$ & Maternal parent & Paternal color & Paternal parent & Hybrid color \\
\hline$\overline{30 \mathrm{~B}}$ & L. ×arkwrightii 'Vesuvius' & White & L. chalcedonica var. alba & $33 \mathrm{~B}$ \\
\hline White & L. chalcedonica var. alba & $44 \mathrm{~B} / 30 \mathrm{~B}^{\mathrm{y}}$ & L. $\times$ haageana mixed hybrids & White \\
\hline $44 \mathrm{~B} / 30 \mathrm{~B}^{\mathrm{y}}$ & L. $\times$ haageana mixed hybrids & White & L. chalcedonica var. alba & $43 \mathrm{~A}$ \\
\hline $37 \mathrm{~A} / 37 \mathrm{~B}$ & L. cognata & $30 \mathrm{~B}$ & L. ×arkwrightii 'Vesuvius' & $30 \mathrm{~A} / 30 \mathrm{~B} / 31 \mathrm{C}$ \\
\hline $30 \mathrm{~B}$ & L. ×arkwrightii 'Vesuvius' & $37 \mathrm{~A} / 37 \mathrm{~B}$ & L. cognata & $30 \mathrm{~A} / 30 \mathrm{~B} / 31 \mathrm{C} / 32 \mathrm{~A} / 32 \mathrm{~B} / 33 \mathrm{~B} / 40 \mathrm{D} / 41 \mathrm{C}$ \\
\hline $37 \mathrm{~A} / 37 \mathrm{~B}$ & L. cognata & $44 \mathrm{~B} / 30 \mathrm{~B}^{\mathrm{y}}$ & L. $\times$ haageana mixed hybrids & $39 \mathrm{~A} / 40 \mathrm{~B} / 40 \mathrm{D} / 43 \mathrm{~A} / 43 \mathrm{~B}$ \\
\hline $44 \mathrm{~B} / 30 \mathrm{~B}^{\mathrm{y}}$ & L. $\times$ haageana mixed hybrids & $37 \mathrm{~A} / 37 \mathrm{~B}$ & L. cognata & $32 \mathrm{~B} / 33 \mathrm{C} / 39 \mathrm{~A} / 40 \mathrm{~A} / 43 \mathrm{C}$ \\
\hline $37 \mathrm{~A} / 37 \mathrm{~B}$ & L. cognata & $44 \mathrm{~B}$ & L. ×haageana 'Molten Lava' & $43 \mathrm{~B} / 43 \mathrm{C} / 40 \mathrm{D}$ \\
\hline 44B & L. ×haageana 'Molten Lava' & $37 \mathrm{~A} / 37 \mathrm{~B}$ & L. cognata & $43 \mathrm{~A}$ \\
\hline $30 \mathrm{~B}$ & L. ×arkwrightii 'Vesuvius' & $44 \mathrm{~B} / 30 \mathrm{~B}^{\mathrm{y}}$ & L. $\times$ haageana mixed hybrids & $33 \mathrm{~B} / 47 \mathrm{~A}$ \\
\hline $44 \mathrm{~B} / 30 \mathrm{~B}^{\mathrm{y}}$ & L. $\times$ haageana mixed hybrids & $30 \mathrm{~B}$ & L. ×arkwrightii 'Vesuvius' & $32 \mathrm{~A} / 33 \mathrm{~A} / 33 \mathrm{~B} / 40 \mathrm{~A}$ \\
\hline $30 \mathrm{~B} / 44 \mathrm{~B}^{\mathrm{y}}$ & L. $\times$ haageana & $37 \mathrm{~A} / 37 \mathrm{~B}$ & L. cognata & $30 \mathrm{~B} / 32 \mathrm{~A} / 33 \mathrm{~B} / 40 \mathrm{D} / 41 \mathrm{C} / 43 \mathrm{~A}$ \\
\hline $44 \mathrm{~B}$ & L. Xhaageana 'Molten Lava' & $30 \mathrm{~A}$ & L. miqueliana & $40 \mathrm{D}$ \\
\hline 44B & L. ×haageana 'Molten Lava' & $44 \mathrm{~B} / 30 \mathrm{~B}^{\mathrm{y}}$ & L. $\times$ haageana mixed hybrids & $32 \mathrm{~A} / 43 \mathrm{~A}$ \\
\hline $30 \mathrm{~B}$ & L. ×arkwrightii 'Vesuvius' & $37 \mathrm{~A} / 37 \mathrm{~B}$ & L. cognata & $30 \mathrm{~A}$ \\
\hline
\end{tabular}

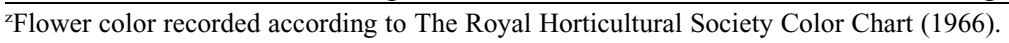

${ }^{\mathrm{y}}$ Flower colors in this species are with many variations.

Table 8. Morphological assessments for six selected interspecific hybrids in Lychnis.

\begin{tabular}{|c|c|c|c|c|c|}
\hline Hybridization $^{z}$ & Corolla diam $(\mathrm{cm})^{\mathrm{y}}$ & Petal length $(\mathrm{cm})^{\mathrm{y}}$ & Petal width $(\mathrm{cm})^{\mathrm{y}}$ & Flower color ${ }^{x}$ & Leaf color $^{\mathrm{x}}$ \\
\hline L. ×arkwrightii 'Vesuvius' & $5.80 \mathrm{a}$ & $2.76 \mathrm{a}$ & $2.14 \mathrm{a}$ & $33 \mathrm{~A}$ & $147 \mathrm{~A}$ \\
\hline L. chalcedonica var. alba & $1.80 \mathrm{c}$ & $0.84 \mathrm{c}$ & $0.70 \mathrm{c}$ & White & $146 \mathrm{~A}$ \\
\hline Hybrids & $3.66 \mathrm{~b}$ & $1.78 \mathrm{~b}$ & $1.82 \mathrm{~b}$ & $33 \mathrm{~B}$ & 147B \\
\hline $\mathrm{LSD}_{0.05}$ & 0.32 & 0.19 & 0.25 & & \\
\hline L. chalcedonica var. alba & $1.80 \mathrm{~b}$ & $0.84 \mathrm{~b}$ & $0.70 \mathrm{~b}$ & White & $146 \mathrm{~A}$ \\
\hline L. $\times$ haageana mixed hybrids & $4.22 \mathrm{a}$ & $1.96 \mathrm{a}$ & $1.78 \mathrm{a}$ & $44 \mathrm{~B}$ & $147 \mathrm{~A}$ \\
\hline hybrids & $1.28 \mathrm{c}$ & $0.58 \mathrm{c}$ & $0.48 \mathrm{c}$ & White/56D & $146 \mathrm{C}$ \\
\hline $\operatorname{LSD}_{0.05}$ & 0.40 & 0.18 & 0.14 & & \\
\hline L. $\times$ haageana mixed hybrids & $4.22 \mathrm{a}$ & $1.96 \mathrm{a}$ & $1.78 \mathrm{a}$ & $44 \mathrm{~B}$ & $147 \mathrm{~A}$ \\
\hline L. chalcedonica var. alba & $1.80 \mathrm{c}$ & $0.84 \mathrm{c}$ & $0.70 \mathrm{c}$ & White & $146 \mathrm{~A}$ \\
\hline Hybrids & $3.00 \mathrm{~b}$ & $1.58 \mathrm{~b}$ & $1.48 \mathrm{~b}$ & $43 \mathrm{~A}$ & $144 \mathrm{~A}$ \\
\hline $\mathrm{LSD}_{0.05}$ & 0.35 & 0.20 & 0.17 & & \\
\hline L. ×arkwrightii 'Vesuvius' & $5.80 \mathrm{a}$ & $2.76 \mathrm{a}$ & $2.14 \mathrm{a}$ & $30 \mathrm{~B}$ & $147 \mathrm{~A}$ \\
\hline L. cognata & $5.22 \mathrm{~b}$ & $2.34 \mathrm{~b}$ & $1.94 \mathrm{a}$ & $37 \mathrm{~A} / 37 \mathrm{~B}$ & 146A/147B \\
\hline Hybrids & $5.56 \mathrm{ab}$ & $2.58 \mathrm{ab}$ & $2.06 \mathrm{a}$ & $40 \mathrm{D} / 30 \mathrm{~A} / 30 \mathrm{~B}$ & 137B \\
\hline $\mathrm{LSD}_{0.05}$ & 0.38 & 0.22 & NS & & \\
\hline L. cognata & $5.22 \mathrm{~b}$ & $2.34 \mathrm{~b}$ & $1.94 \mathrm{~b}$ & $37 \mathrm{~A} / 37 \mathrm{~B}$ & $146 \mathrm{~A} / 147 \mathrm{~B}$ \\
\hline L. ×haageana 'Molten Lava' & $4.60 \mathrm{c}$ & $2.24 \mathrm{~b}$ & $2.02 \mathrm{~b}$ & 44B & $147 \mathrm{~A} / 147 \mathrm{~B}$ \\
\hline hybrids & $6.00 \mathrm{a}$ & $2.76 \mathrm{a}$ & $2.74 \mathrm{a}$ & $43 \mathrm{~B} / 43 \mathrm{C} / 40 \mathrm{D}$ & $146 \mathrm{~A} / 147 \mathrm{~A}$ \\
\hline $\mathrm{LSD}_{0.05}$ & 0.40 & 0.18 & 0.31 & & \\
\hline L. ×haageana 'Molten Lava' & $4.60 \mathrm{~b}$ & $2.24 \mathrm{~b}$ & $2.02 \mathrm{~b}$ & 44B & $147 \mathrm{~A} / 147 \mathrm{~B}$ \\
\hline L. cognata & $5.22 \mathrm{a}$ & $2.34 \mathrm{~b}$ & $1.94 \mathrm{~b}$ & $37 \mathrm{~A} / 37 \mathrm{~B}$ & 146A/147B \\
\hline Hybrids & $5.66 \mathrm{a}$ & $2.64 \mathrm{a}$ & $2.38 \mathrm{a}$ & $\begin{array}{c}33 \mathrm{~A} / 33 \mathrm{~B} / 34 \mathrm{~A} \\
43 \mathrm{~A} / 43 \mathrm{~B} / 43 \mathrm{C}\end{array}$ & 146A/147B \\
\hline$\underline{\mathrm{LSD}_{0.05}}$ & 0.58 & 0.27 & 0.34 & & \\
\hline
\end{tabular}

${ }^{\mathrm{z}}$ Maternal plant followed by paternal plant.

y Different letters in one column indicate means significantly different at $P \leq 0.05, \mathrm{n}=5$.

${ }^{x}$ Flower and leaf color recorded according to The Royal Horticultural Society Color Chart (1966).

LSD = least significant difference; NS = nonsignificant.

and L. $\times$ haageana mixed hybrids $\times$ L. chalcedonica var. alba, progeny flower color was more similar to the maternal parent.

Intergeneric crosses. Because of crossincompatibility, crossing combinations between Lychnis spp. and Silene spp. had the lowest number of seed setting flowers and seeds. Of 290 crosses made, only 39 set seed
(Tables 9 and 10). Partial compatible crossing combinations were made from $L$. chalcedonica $\times S$. armeria, L. cognate $\times S$. plankii and its reciprocal cross, $L$. cognate $\times S$. pendula, $S$. plankii $\times$ L. miqueliana, $L$. $\times$ arkwrightii 'Vesuvius' $\times S$. armeria, $L$. $\times$ arkwrightii 'Vesuvius' $\times S$. pendula, $S$. plankii $\times$ L. ×arkwrightii 'Vesuvius', $L$. $\times$ haageana mixed hybrids $\times S$. armeria, L. $\times$ haageana mixed hybrids $\times S$. pendula, L. $\times$ haageana 'Molten Lava' $\times S$. pendula, L. wilfordii $\times S$. armeria, and L. wilfordii $\times$ $S$. pendula. Among intergeneric crossing combinations, seed set rates varied. No seeds germinated; thus, embryo rescue techniques with immature seed culture would likely 
Table 9. Flower pollination, seed set, and germination for intergeneric crosses as seed parent between Lychnis and Silene.

\begin{tabular}{|c|c|c|c|c|c|c|}
\hline Seed parent & $\begin{array}{l}\text { No. of species } \\
\text { mating }\end{array}$ & $\begin{array}{c}\text { No. of cross- } \\
\text { pollinated flowers }\end{array}$ & $\begin{array}{l}\text { Percent of flowers } \\
\text { setting seed }\end{array}$ & $\begin{array}{l}\text { Avg seed no. } \\
\text { per capsule }\end{array}$ & $\begin{array}{l}\text { Total no. } \\
\text { of seeds }\end{array}$ & $\begin{array}{c}\text { Percent } \\
\text { germination }\end{array}$ \\
\hline L. chalcedonica 'Burning Love' & 1 & 6 & 0.0 & 0 & 0 & 0.0 \\
\hline L. chalcedonica "White' & 2 & 10 & 0.0 & 0 & 0 & 0.0 \\
\hline Subtotal for $L$. chalcedonica & 2 & 33 & 9.1 & 12 & 37 & 0.0 \\
\hline L. ×arkwrightii 'Orange Genome' & 1 & 3 & 0.0 & 0 & 0 & 0.0 \\
\hline L. flos-cuculi 'White Robin' & 1 & 5 & 0.0 & 0 & 0 & 0.0 \\
\hline L. $\times$ haageana mixed hybrids & 4 & 34 & 176 & 86 & 45 & 0.0 \\
\hline L. ×haageana 'Molten Lava' & 1 & 3 & 100.0 & 14 & 41 & 0.0 \\
\hline L. cognata & 3 & 25 & 28.0 & 12 & 82 & 0.0 \\
\hline S. armeria & 7 & 77 & 0.0 & 0 & 0 & 0.0 \\
\hline S. delavayi & 1 & 1 & 0.0 & 0 & 0 & 0.0 \\
\hline S. plankii & 4 & 21 & 52.4 & 36 & 394 & 0.0 \\
\hline S. pendula & 6 & 35 & 0.0 & 0 & 0 & 0.0 \\
\hline Total for genus Silene & 7 & 134 & 8.2 & 36 & 394 & 0.0 \\
\hline
\end{tabular}

Table 10. Flower pollination, seed set, and germination for intergeneric crosses as pollen parent between Lychnis and Silene.

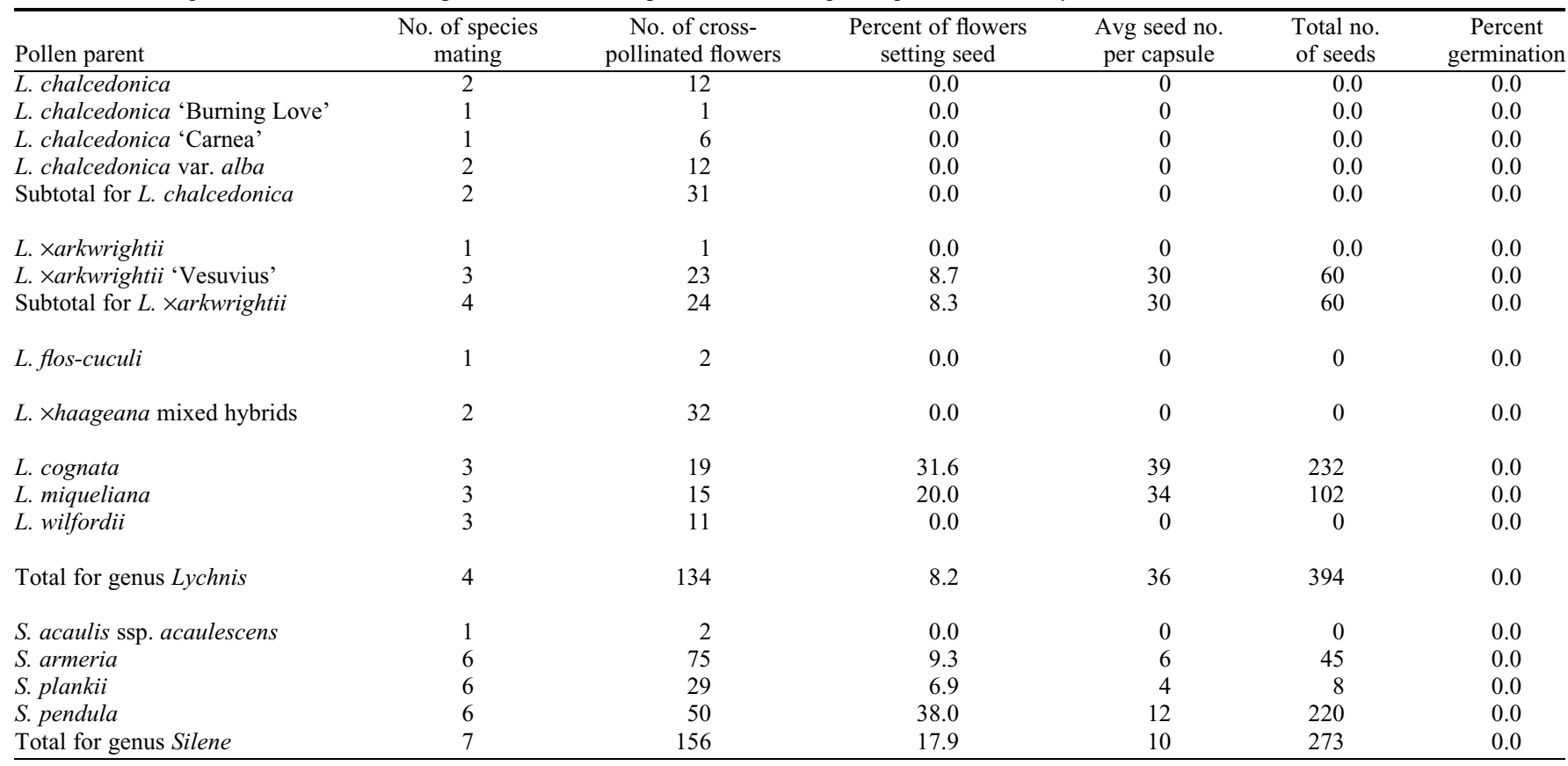

have to be used to produce seedlings. Again, seed set differences in reciprocal crosses helped to partially overcome interspecific cross-incompatibility between two genera.

Selfing rate. According to our observation, L. chalcedonica and L. flos-cuculi have a very high selfing rate. Lychnis wilfordii yielded a very low selfing rate in the greenhouse despite producing a lot of pollen. No hybrid plants were obtained from $L$. wilfordii, yet the profuse flowering makes it a potential parent for ornamental breeding through embryo rescue.

Breeding potentials. The common name of the family Caryophyllaceae is carnation family or pink family; however, pink color is not a prevailing color in Lychnis species used in the hybridizations. Two species, $L$. miqueliana and L. cognata, are pink with relatively large flowers making them good potential parents, but both had weak stems when cultivated in the greenhouse. Lychnis miqueliana did not readily produce hybrids with other species. The interspecific hybridizations between L. cognata and other Lychnis species were successful. Six hybrids generated from L. cognata were pink (Table 7). The stem color of the hybrids between L. Xarkwrightii 'Vesuvius' and L. cognata was partially inherited from 'Vesuvius' showing reddish internodes. The stems were stronger than L. cognata. Lychnis cognata proved to be a good parent for crossing for its promising interspecific cross-compatibility and pink flower color. In this study, at least 11 interspecific hybrids with rich variations in flower color, stem type, stem color, and leaf color were generated without the need for embryo rescue. Six promising hybrids were selected according to their attractive ornamental phenotypes and morphology (Table 8). To expand genetic variations for ornamental flower breeding programs using Lychnis and Silene, immature seed culture protocols, phenotype selection, fertility 
status, and propagation protocols for hybrids need to be investigated further.

\section{Discussion}

Hybridization at intraspecific, interspecific, and intergeneric levels is commonly practiced in breeding programs to combine desirable traits. Our results demonstrated that mature seeds and normal plants can be produced from intraspecific hybridization within L. chalcedonica and L. Xhaageana species, confirming results by Nakano et al. (2013). In their study, Nakano et al. (2013) made cross-pollinations among eight species with the same chromosome number $(2 \mathrm{n}=2 \mathrm{x}=24)$ in Lychnis, including $L$. chalcedonica, $L$. coronata, L. fulgens, L. gracillima, L. kiusiana, L. miqueliana, L. sieboldii, and L. wilfordii; based on the average value of each species, $L$. coronata yielded both the lowest seed set $(8.3 \%)$ and seed number with four per capsule. Seed set for the other seven species ranged from $50.6 \%$ to $81.5 \%$, and seed number per capsule ranged from seven to 55. However, only immature seeds were obtained from these interspecific crossing combinations 4 weeks after pollination. By immature seed culture on half-strength Murashige and Skoog medium without plant growth regulators, of a total of 26 crosses that produced seeds, seeds from 11 crosses germinated in 3 months, and only one of them produced seedlings. Compared with the previously mentioned report, we used different species and many cultivars in some species to test crossing compatibility for interspecific crosses in Lychnis. Also, the number of crosses including reciprocal crosses in our study was higher. Based on our study, high seed set and germination percentages were observed from the interspecific crossing combination among $L$. cognate, L. ×arkwrightii, and L. Xhaageana with no cross-incompatible barriers existing between them. For most of the other interspecific crosses, our results were similar to Nakano et al.'s report (Nakano et al., 2013). For those crosses that only have immature seeds, embryo rescue methods need to be developed to produce hybrid plants. Also, the fertility and propagation procedure of interspecific hybrids should be investigated further.

Although all plants have the same chromosome number, cross-compatibility and hybrid seed germination between Lychnis and Silene were significantly lower than those between species in the genus Lychnis. The production of $F_{1}$ hybrids showed some indication that Lychnis and Silene are closely related. This breeding evidence seems to support the conclusion that Lychnis and Silene are two genera instead of one genus classification (Rabeler, 1992). However, considering the large number of species in Silene and their broad distribution, the generic delimitation of Silene still needs to be studied in detail.

Because a significant number of crosses in a blooming season was performed with limited personnel in this study, hand hybridization without emasculation was conducted to save time and labor. Crosses are sometimes made without emasculation such as Yang et al. (2010) who conducted intergeneric hybridization without emasculation between Opisthopappus taihangensis (Ling) C. Shih. and Chrysanthemum lavandulifolium (Fisch. ex Trautv.). From our observation in the greenhouse, Lychnis is protandry, which facilitates non-emasculation if large hybrid populations are wanted with limited personnel. Genotype fingerprinting tools could be used to verify hybridity (Nakano et al., 2013). Pollen storage and embryo rescue techniques would facilitate a greater number of intraspecific, interspecific, and intergeneric crosses and hybrids.

\section{Literature Cited}

Desfeux, C. and B. Lejeune. 1996. Systematics of euromediterranean Silene (Caryophyllaceae): Evidence from a phylogenetic analysis using ITS sequences. Cr. Acad. Sci. Iii-Vie 319:351358.

Godo, T., J. Miyazaki, S. Kuwayama, S. Ogita, Y. Kato, M. Nakano, and M. Nakata. 2009. Interspecific hybridization between triploid Senno (Lychnis senno Siebold et Zucc.,
Caryophyllaceae) and allied taxa of the genus Lychnis. Plant Biotechnol. 26:301305 .

Kruckeberg, A. 1962. Intergeneric hybrids in the lychnideae (Caryophyllaceae). Brittonia 14: 311-321.

Mayol, M. and J.A. Rossello. 2006. Hybridization studies in Silene subgen. petrocoptis (Caryophyllaceae). Folia Geobot. 41:203-212.

Melzheimer, V. 1988. Silene. In: Rechinger, K.H. (ed.). Flora iranica Lfg. Akademische Druck- \& Verlagsanstalt, Graz. 163:341-508.

Nakano, M., S. Kuwayama, E. Oka, M. Asano, D. Han, and T. Godo. 2013. Cross-compatibility in interspecific hybridization of Lychnis (Caryophyllaceae) and characterization of interspecific hybrids between $L$. fulgens and L. sieboldii. J. Jpn. Soc. Hort. Sci. 82:57-62.

Negrutiu, I., B. Vyskot, N. Barbacar, S. Georgiev, and F. Moneger. 2001. Dioecious plants. A key to the early events of sex chromosome evolution. Plant Physiol. 127:1418-1424.

Oxelman, B., M. Lidén, R.K. Rabeler, and M. Popp. 2000. A revised classification of the tribe Sileneae (Caryophyllaceae). Nord. J. Bot. 20:743-748.

Popp, M., A. Gizaw, S. Nemomissa, J. Suda, and C. Brochmann. 2008. Colonization and diversification in the African 'Sky Islands' by Eurasian Lychnis L. (Caryophyllaceae). J. Biogeogr. 35:1016-1029.

Rabeler, R.K. 1992. Proposal to conserve 2490 Silene L. against Lychnis L. (Caryophyllaceae). Taxon 41:126-128.

Royal Horticultural Society. 1966. Royal Horticultural Society colour chart. Royal Hort. Soc., London, and Flower Council of Holland, Leiden.

Walters, S.M. 1989. Silene; Lychnis, p. 179-182. In: Walters, S.M., J.C.M. Alexander, A. Brady, C.D. Brickell, J. Cullen, P.S. Green, V.H. Heywood, V.A. Matthews, N.K.B. Robson, P.E. Yeo, and S.G. Knees (eds.). The European garden flora, 3. Cambridge University Press, Cambridge, UK.

Wilson, G.B., J. Wright, P. Lusby, W.J. Whittington, and R.N. Humphries. 1995. Lychnis viscaria L (Viscaria vulgaris Bernh). J. Ecol. 83:10391051.

Yang, D.Y., X. Hu, Z.H. Liu, and H.E. Zhao 2010. Intergeneric hybridizations between Opisthopappus taihangensis and Chrysanthemum lavandulifolium. Sci. Hort. 125:718723. 